\title{
ON A THEOREM OF CAMBERN
}

\author{
H. U. HESS
}

\begin{abstract}
It is shown that Cambern's generalized Banach-Stone theorem for spaces of continuous functions on compact sets cannot be extended to the case of spaces of continuous affine functions on simplexes.
\end{abstract}

Introduction. The present paper is concerned with the question whether Cambern's theorem on isomorphisms with small bound for spaces $C(X)$ of continuous real functions on compact sets $X$ can be generalized to spaces $A(K)$ of continuous affine functions on compact simplexes $K$. The theorem of Cambern [1], in question, is the following:

If $X$ and $Y$ are compact and if there is a linear isomorphism $\phi$ of $C(X)$ onto $C(Y)$ with $\|\phi\| \cdot\left\|\phi^{-1}\right\|<2$ then $X$ and $Y$ are homeomorphic. ${ }^{1}$

In terms of continuous affine functions, this statement can be rephrased as follows:

If $K_{1}$ and $K_{2}$ are Bauer simplexes and if there is a linear isomorphism $\phi$ of $A\left(K_{1}\right)$ onto $A\left(K_{2}\right)$ with $\|\phi\| \cdot\left\|\phi^{-1}\right\|<2$ then the sets of extreme points $\operatorname{ex}\left(K_{1}\right)$ and $\operatorname{ex}\left(K_{2}\right)$ are homeomorphic.

In this note, we give a negative answer to the question whether or not this result holds for arbitrary simplexes $K_{1}$ and $K_{2}$, by proving:

Theorem. For each $\alpha \in$ ]0, 1[ there exist two simplexes $K_{1}$ and $K_{2}$ such that $\operatorname{ex}\left(K_{1}\right)$ and $\operatorname{ex}\left(K_{2}\right)$ are not homeomorphic and such that there exists a linear isomorphism $\phi$ of $A\left(K_{1}\right)$ onto $A\left(K_{2}\right)$ with $\|\phi\| \cdot\left\|\phi^{-1}\right\|<1+\alpha$.

As the proof of the theorem will show, here $K_{1}$ can be chosen to be a Bauer simplex. Note that $A\left(K_{1}\right)$ and $A\left(K_{2}\right)$ cannot possibly be isometric if $\operatorname{ex}\left(K_{1}\right)$ and $\operatorname{ex}\left(K_{2}\right)$ are not homeomorphic and if $K_{1}$ and $K_{2}$ are simplexes (see [2, p. 169]).

Notations. Let $\bar{N}$ denote the one point compactification of the discrete space $\mathbf{N}$ of positive integers. For every $r \in] 0$, 1[ we define

$$
A_{r}:=\{f \in C(\bar{N}) \mid f(\infty)=r \cdot f(1)+(1-r) \cdot f(2)\}
$$

Received by the editors January 4, 1978 and, in revised form, February 8, 1978.

AMS (MOS) subject classifications (1970). Primary 46A40, 46B99, 46E15.

${ }^{1}$ Cambern [1] also proved that this result remains true if $X$ and $Y$ are locally compact and $C(X)$ (resp. $C(Y)$ ) denotes the space of continuous functions on $X$ (resp. $Y$ ) which are zero at infinity. 
and

$$
K_{r}:=\left\{m \in A_{r}^{*} \mid m \geqslant 0, m(1)=1\right\}
$$

LEMMA 1. For every $r \in] 0,1\left[\right.$ the $w^{*}$-compact convex set $K_{r}$ is a simplex.

Proof. We know that for every $r \in$ ]0, 1[ the Choquet boundary for $A_{r}$ coincides with $\mathbf{N}$. Besides, we see that the only measures on $\bar{N}$ which annihilate $A_{r}$ are the real multiples of

$$
\varepsilon_{\infty}-r \cdot \varepsilon_{1}-(1-r) \cdot \varepsilon_{2}
$$

and these are not supported by $\mathbf{N}$; that is the only annihilating boundary measure for $A_{r}$ is 0 , so $K_{r}$ is a simplex.

LEMMA 2. For every $r \in] 0,1\left[\right.$ there exists a linear isomorphism $\phi_{r}$ of $A_{r}$ onto $C(\bar{N})$ such that

$$
\left\|\phi_{r}(f)\right\| \leqslant\|f\| \leqslant(2 / r-1) \cdot\left\|\phi_{r}(f)\right\| \text { for all } f \in A_{r} .
$$

Proof. We define $\phi_{r}: A_{r} \rightarrow C(\bar{N})$ by

$$
\left(\phi_{r}(f)\right)(i):=\left\{\begin{array}{l}
f(i+1) \quad \text { if } i \in \mathbf{N} \\
f(\infty) \text { if } i=\infty .
\end{array}\right.
$$

The linear map $\phi_{r}$ is one to one since the equality $\phi_{r}(f)=0$ implies: $f(i+1)=0$ for all $i \in \mathbf{N}$ and

$$
0=f(\infty)=r \cdot f(1)+(1-r) \cdot f(2)=r \cdot f(1),
$$

from which the equality $f=0$ follows.

$\phi_{r}$ is surjective too, for if we choose $g \in C(\bar{N})$ and define

$$
h(i):=\left\{\begin{array}{l}
(1 / r) \cdot(g(\infty)-(1-r) \cdot g(1)) \text { if } i=1, \\
g(i-1) \text { if } \infty>i \geqslant 2 \\
g(\infty) \text { if } i=\infty
\end{array}\right.
$$

then $h \in A_{r}$ and $\phi_{r}(h)=g$.

Now, choose $f \in A_{r}$. We see immediately that $\left\|\phi_{r}(f)\right\|<\|f\|$. To show the inequality $\|f\| \leqslant(2 / r-1) \cdot\left\|\phi_{r}(f)\right\|$ we may suppose that $\|f\|>\left\|\phi_{r}(f)\right\|$, because otherwise $\|f\| \leqslant\left\|\phi_{r}(f)\right\| \leqslant(2 / r-1) \cdot\left\|\phi_{r}(f)\right\|$. Hence we obtain the following inequality

$$
\begin{aligned}
\|f\| & =\sup \{\mid f(i) \| i \in \mathbf{N}\} \\
& =\max (\sup \{\mid f(i) \| i \in \mathbf{N} \backslash\{1\}\},|f(1)|) \\
& =\max \left(\left\|\phi_{r}(f)\right\|,|f(1)|\right)=|f(1)| \\
& =(1 / r) \cdot|f(\infty)-(1-r) \cdot f(2)| \leqslant(1 / r) \cdot\left(\left\|\phi_{r}(f)\right\|+(1-r) \cdot\left\|\phi_{r}(f)\right\|\right) \\
& =(2 / r-1) \cdot\left\|\phi_{r}(f)\right\| .
\end{aligned}
$$

This proves the assertion of Lemma 2. 
Proof of THE theorem. Choose $\alpha \in] 0,1\left[\right.$ and put $r_{\alpha}=2 /(2+\alpha)$. Then, according to Lemma 2, there is a linear isomorphism $\phi_{r_{\alpha}}$ of $A_{r_{\alpha}}$ onto $C(\bar{N})$ with $\left\|\phi_{r_{a}}\right\| \cdot\left\|\phi_{r_{a}}^{-1}\right\| \leqslant\left(2 / r_{\alpha}-1\right)=(1+\alpha)$. Therefore, by the usual identification of $A_{r_{\alpha}}$ with $A\left(K_{r_{a}}\right)$ and of $C(\bar{N})$ with $A(K)$, where $K$ denotes the set of all probability measures on $\bar{N}$, we obtain the following result: For every $\alpha \in] 0,1\left[\right.$ there exist $\left.r_{\alpha} \in\right] 0,1\left[\right.$ and a linear isomorphism $\phi_{r_{\alpha}}$ of $A\left(K_{r_{\alpha}}\right)$ onto $A(K)$ such that $\left\|\phi_{r_{\alpha}}\right\| \cdot\left\|\phi_{r_{\alpha}}^{-1}\right\| \leqslant 1+\alpha$. Beside this, we know by Lemma 1 that $K_{r_{a}}$ is a simplex. Since ex $\left(K_{r_{a}}\right)($ resp. ex $(K))$ can be identified with $\mathbf{N}$ (resp. $\bar{N}), \operatorname{ex}\left(K_{r_{\alpha}}\right)$ and ex $(K)$ are not homeomorphic. This completes the proof of the theorem.

ACKNowledgement. I would like to thank Professor D. Kölzow for raising the question of this paper and for helpful conversations.

\section{REFERENCES}

1. M. Cambern, On isomorphisms with small bound, Proc. Amer. Math. Soc. 18 (1967), 1062-1066.

2. A. Lazar, Affine products of simplexes, Math. Scand. 22 (1968), 165-175.

FACHBEREICH MATHEMATIK, UNIVERSITÄT REgenSBURG, UNIVERSITÄTSSTRASSE 31, 8400 REgensburg, West Germany 